\title{
Kinetic and energetic insights in the dissipative non-equilibrium operation of an autonomous light-powered supramolecular pump
}

Stefano Corra, ${ }^{\ddagger, 1,2}$ Marina Tranfic Bakic, ${ }^{\ddagger, 1,2}$ Jessica Groppi, ${ }^{1}$ Massimo Baroncini, ${ }^{1,4}$ Serena Silvi, ${ }^{1,3}$ Emanuele Penocchio ${ }^{5}$, Massimiliano Esposito ${ }^{5}$, and Alberto Credi ${ }^{1,2, *}$

${ }^{1}$ CLAN-Center for Light Activated Nanostructures, Istituto ISOF-CNR, via Gobetti 101, 40129 Bologna, Italy

2 Dipartimento di Chimica Industriale "Toso Montanari", Università di Bologna, viale del Risorgimento 4, 40136 Bologna, Italy

${ }^{3}$ Dipartimento di Chimica "G. Ciamician", Università di Bologna, via Selmi 2, 4126 Bologna, Italy

${ }^{4}$ Dipartimento di Scienze e Tecnologie Agro-alimentari, Università di Bologna, viale Fanin 44, 40127 Bologna, Italy

${ }^{5}$ Department of Physics and Materials Science, University of Luxembourg, L-1511 Luxembourg City, G. D. Luxembourg

$\ddagger$ these authors contributed equally to this work

\begin{abstract}
Natural and artificial autonomous molecular machines operate by constantly dissipating energy coming from an external source to maintain a non-equilibrium state. The in-depth study of these dissipative states is highly challenging as they exist only as long as energy is provided. Here we report on the detailed physicochemical characterization of the dissipative operation of a supramolecular pump transducing light energy into chemical energy by shifting the equilibrium of self-assembly reactions. The composition of the system under light irradiation was followed in realtime by ${ }^{1} \mathrm{H}$ NMR and parameters such as the dissipation and the energy storage at the steady state were extracted for four different irradiation intensities. For the first time in an artificial system, we quantitatively probed the relationship between the light energy input and the deviation of the dissipative state from thermodynamic equilibrium. Our results also provide a testing ground for newly developed theoretical models.
\end{abstract}


Natural and artificial molecular machines, such as autonomous molecular motors and pumps, harvest energy from chemical reactions $s^{1,2,3,4,5,6,7,8,9,10}$ or from processes involving light absorption ${ }^{11,12,13,14,15,16,17,18,19,20}$ to sustain a non-equilibrium state (Fig. 1a). ${ }^{21,22,23,24,25,26}$

The determination of the speciation in the out-of-equilibrium state is highly desirable as several thermodynamic and kinetic parameters of the machine operation could be extracted. The characterization of dissipative states, however, is extremely challenging as it requires any analysis to be performed during operation, that is in the presence of the fuel-to-waste conversion. ${ }^{27,28,29}$
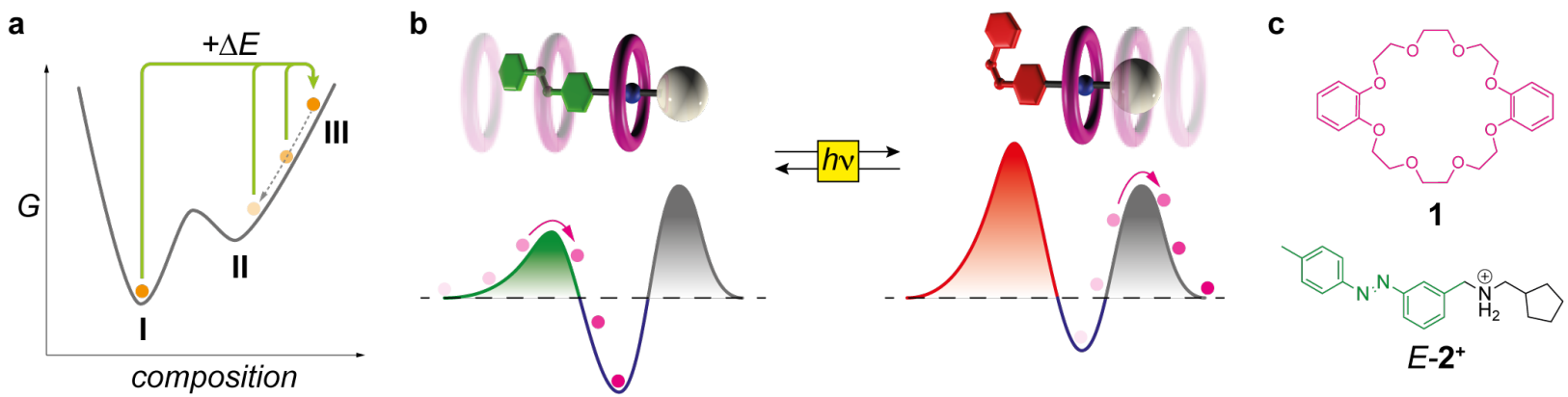

Figure 1. Simplified energy diagrams of operation of the pump and molecular structures of the components. a) Idealized landscape of the total Gibbs free energy of a chemical system, showing the global thermodynamic minimum that corresponds to the thermal equilibrium (I), a local equilibrium state (II), and a dissipative non-equilibrium state (III). State III exists only if energy is continuously supplied to the system (solid green arrows) to prevent its relaxation to state II (dashed gray arrow). b) Schematic representation of the relative unidirectional translation of molecular ring and axle components triggered by light, i.e., a supramolecular pump powered by light. The simplified potential energy curves (free energy versus ring-axle distance) corresponding to the two structures illustrate the energy ratchet mechanism that rectifies the Brownian motion of the ring. c) Molecular structure of the components of the supramolecular pump of the present study: the DB24C8 macrocycle 1 and the nonsymmetric molecular axle E-2 ${ }^{+}$.

We previously reported on a series of light-powered supramolecular pumps based on a pseudorotaxane complex architecture. The operational principle lays on the reversible photoisomerization of the azobenzene moiety of the axle which enables a flashing energy ratchet mechanism (Fig. 1b). Under continuous irradiation, the crown ether macrocycle undergoes autonomous and directional cycling between the assembled and disassembled states. ${ }^{15,30,31}$ The pump dissipative operation was proven comparing the thermodynamic and kinetic data for the two - $E$ and $Z$ - isomers, as well as by ${ }^{1} \mathrm{H}$ NMR irradiating the sample solution inside the NMR probehead. ${ }^{30,31,32}$ Specifically, this technique allows for the real-time monitoring of the system composition at the dissipative steady state. Building upon these results, we report on the physicochemical characterization of the dissipative steady state and operation cycle of a secondgeneration molecular pump. The cycling rate, quantum yield, energy storage, and power conversion efficiency were derived from the experimental concentrations at different incident photon flows. Moreover, for the first time in an artificial system, we probed a relationship between the light energy input and the deviation of the dissipative state from the equilibrium composition. 


\section{Structure and operation of the molecular pump.}

The supramolecular pump is composed of a dibenzo[24]crown-8 (DB24C8) ether macrocycle (1) and a molecular axle $\left(2^{+}\right)$, which comprises a secondary ammonium recognition site for 1 flanked by a photoswitchable azobenzene gate and a cyclopentyl 'pseudostopper' (Fig. 1c). Briefly, in acetonitrile the macrocycle encircles the secondary ammonium station of the axle to form a stable hydrogen-bonded rotaxane-type complex for both the $E$ and $Z$ isomers of $2^{+}$. The $E$ complex is slightly more stable than the $Z$ complex $\left(K_{a}{ }^{E}>K_{a}{ }^{Z}\right)$ and its formation occurs almost exclusively by slippage of the ring over the $E$-azobenzene moiety (Table 1). The non-photoactive pseudostopper was selected to ensure that the formation of the $Z$ complex occurs almost exclusively by slippage of the ring over the cyclopentyl moiety. ${ }^{30,31}$

The $E \rightarrow Z$ isomerization of the axle has therefore two key consequences: (i) the destabilization of the threaded complex, and (ii) the increase of the activation energy for the slippage of the ring over the azobenzene extremity. ${ }^{30,31,33}$ As a result, light induced unidirectional transport of the rings over the pump occurs according to an energy ratchet mechanism (Fig. 1b). As both $E$ and $Z$ isomers of azobenzene are photoreactive and exhibit highly overlapped absorption spectra, photons of the same wavelength can trigger both $E \rightarrow Z$ and $Z \rightarrow E$ isomerization. The system is, thus, able to repeat the cycle autonomously under constant illumination, reaching and maintaining a stationary non-equilibrium state dissipating light energy.

Table 1. Thermodynamic and kinetic parameters in air-equilibrated $\mathrm{CD}_{3} \mathrm{CN}$ at $298 \mathrm{~K}$ for the rotaxane-type complexes formed between $\mathbf{1}$ and either $E-\mathbf{2}^{+}$or $Z-\mathbf{2}^{+}$.[a]

\begin{tabular}{|c|c|c|c|}
\hline & $\begin{array}{c}K_{a} \\
\left(\mathrm{~mol} \mathrm{~L}^{-1}\right)^{[\mathrm{b}]}\end{array}$ & $\begin{array}{c}k_{\text {in }} \\
\left(\mathrm{L} \mathrm{mol}^{-1} \mathrm{~s}^{-1}\right)^{[\mathrm{c}]}\end{array}$ & $\begin{array}{c}k_{\text {out }} \\
\left(\mathrm{s}^{-1}\right)^{[\mathrm{d}]}\end{array}$ \\
\hline $1+E-2^{+}$ & $230 \pm 30$ & $16 \pm 3^{[\mathrm{e}]}$ & $0.07^{[f]}$ \\
\hline $1+Z-2^{+}$ & $115 \pm 35^{[\mathrm{g}]}$ & $(3.1 \pm 0.8) \times 10^{-2[\mathrm{~h}]}$ & $(2.7 \pm 0.5) \times 10^{-4[h]}$ \\
\hline \multicolumn{4}{|c|}{ 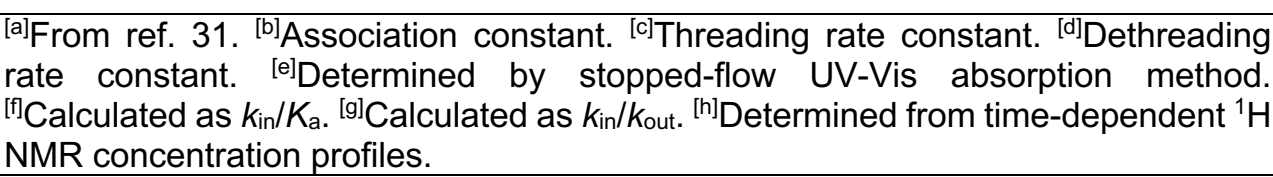 } \\
\hline
\end{tabular}

The operation of the molecular pump can be described with the reaction network depicted in Fig. 2. Two thermal processes (self-assembly, numbered 1 and 3) and two photochemical processes ( $E / Z$ isomerization, numbered 2 and 4 ) are involved in the cycle and only the former ones must satisfy microscopic reversibility, as photochemical processes result from reaction mechanisms involving excited states of the molecules. ${ }^{34,35,36,37}$ Since the photoisomerization quantum yields and molar absorption coefficients of the free and complexed axle are almost identical (Table S2), they exhibit essentially the same E/Z composition at the PSS, that is $K_{\mathrm{hv}}{ }^{\mathrm{u}}=K_{\mathrm{hv}}{ }^{\mathrm{c}}{ }^{38}$ Conversely, the equilibrium constants for the self-assembly processes are different, specifically $K_{a}{ }^{E}>K_{a}{ }^{Z} \cdot{ }^{31}$ As a consequence, under irradiation detailed balance in this reaction network cannot be fulfilled and the cycle is travelled clockwise with a non-zero net rate. ${ }^{31,39}$ The 
ability of the system to cycle preferentially clockwise with respect to anticlockwise - that is its kinetic asymmetry - is quantified by the ratcheting constant $\left(K_{r}\right)$, defined as the ratio between the clockwise and anticlockwise rates. ${ }^{40,41}$ For our system under irradiation, $K_{r}$ is well approximated by the ratio $K_{a}{ }^{E} / K_{a}{ }^{Z}=2$ meaning that, on average, the system loops anticlockwise once every two cycles in the clockwise direction (see the SI).

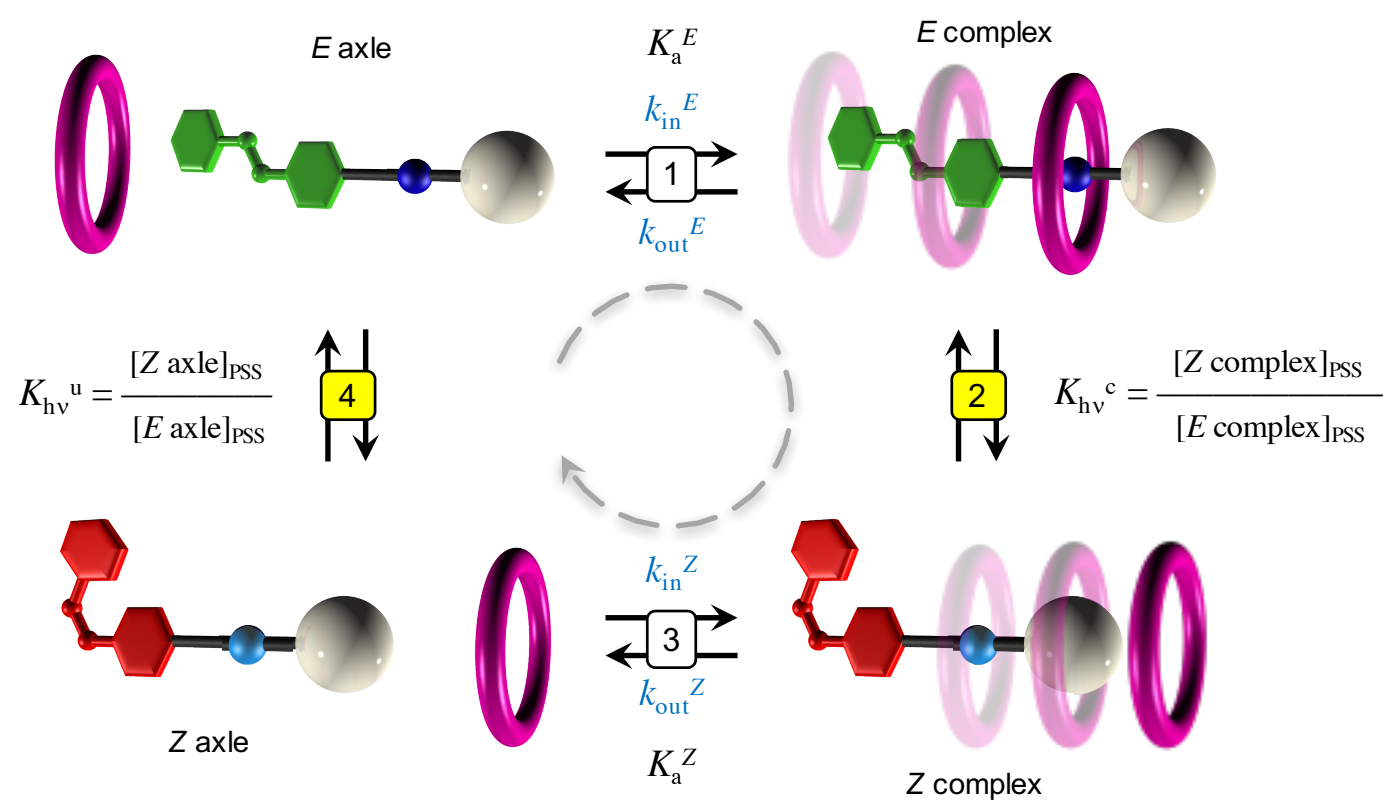

Figure 2. Operative reaction network of the supramolecular pump. Thermal self-assembly equilibria ( 1 and 3 ) and photochemical isomerization reactions ( 2 and 4 ). The dashed circular arrow indicates the net direction of travelling of the cycle. Conventionally all the parameters are positive for the reactions read from left to right and from top to bottom.

\section{Study of the light-dependent dissipative non-equilibrium regime.}

A typical feature of dissipative systems is that a fraction of the energy input is employed to shift concentrations away from equilibrium values and is, thus, stored as free energy into the system. $^{23,13,42,43}$ In fact, in a closed cycle of reactions when detailed balance is not fulfilled, the cycling net rate must be equal for all steps and non-zero $\left(v_{c y} \equiv v_{1}=v_{2}=-v_{3}=-v_{4} \neq 0\right) .{ }^{34}$ To satisfy this condition, the concentration of the species under operation must adjust to a level which is different from that of equilibrium. This is exactly what is observed for the cycle of the supramolecular pump (Fig. 2). ${ }^{31}$

Since the rates of the individual photochemical reactions $\left(v_{2}\right.$ and $\left.v_{4}\right)$ are dictated by the photon flow, variation of the latter will modify the overall cycling rate. Therefore, the self-assembly steps ( 1 and 3 ) must adapt their net rate of reaction to the new cycling rate adjusting the concentration of reagents and products to a different level. As a consequence, for reactions 1 and 3 a larger deviation from the equilibrium concentrations is expected at a higher photon flow. In turns, this means that, also in a light driven system, the amount of stored energy should be 
correlated to the amount of energy provided by the external reservoir. ${ }^{42,37}$

To probe this hypothesis it is crucial to measure the speciation in real time upon operation of the pump. In situ irradiation in the NMR probehead, as demonstrated recently, is perfectly suited for this task. ${ }^{31,32}$ We employed an optical fibre to deliver nearly monochromatic UV-light $\left(\lambda_{\text {irr }}=365\right.$ $\pm 5 \mathrm{~nm}$ ) to the solution inside the NMR tube (Fig. S2). The monochromatic setup is advantageous because (i) the behaviour of the system can be appropriately described using spectroscopic and photochemical parameters independently measured for the pump components (Table S2) and (ii) the photon flow can be accurately determined by chemical actinometry. Both these points are fundamental to perform quantitative numerical simulations to support the pump operating cycle.

Typically, an equimolar mixture of $E-2^{+}$and $1(8.9 \mathrm{mM})$ was equilibrated in the dark and then irradiated with full-power $365 \mathrm{~nm}$ light (photon flow, $4.4 \times 10^{-8}$ Einstein s $\mathrm{s}^{-1}$ ) until a constant Z/E composition is reached (35 $\mathrm{min}$ ). During this time, the E-configured axle and complex are photoconverted to the corresponding $Z$ species faster than the disassembly of the $Z$ complex. Thus, the $Z$ species are produced in relative concentrations that are not consistent with $K_{\mathrm{a}}{ }^{Z}$; specifically, the $Z$ complex is kinetically trapped in a concentration higher than its equilibrium value. In a first instance, after reaching the photostationary state (PSS), the irradiation was turned off and spectra were acquired over time. In the dark, a decrease in the concentration of the $Z$ complex, accompanied by a corresponding increase in the concentration of $Z$ axle, was observed, as 1 dethreads to satisfy the equilibrium condition. After about two hours, (local) equilibrium is reached with a decrease in the $Z$ complex concentration of about $10 \%$ (Fig. 3a). ${ }^{44}$ It is important to note that the thermal $Z \rightarrow E$ isomerization during this interval is negligible.

In a second instance, after reaching the PSS the irradiation was continued with the same photon flow $\left(4.4 \times 10^{-8}\right.$ Einstein $\left.\mathrm{s}^{-1}\right)$, or with a flow attenuated to 50,25 , or $10 \%$ of the initial value (Fig. S2). In all cases, new PSS were reached within about one hour in which the concentration of the $Z$ complex is higher than the value reached in the dark (Fig. 3b-e). On the other hand, the concentration of the $Z$ axle is lower than that in the dark (Fig. S4). These results confirm that the system reaches a dissipative non-equilibrium steady state in which the $Z$ complex is kinetically accumulated at the expenses of the $Z$ axle. ${ }^{31}$ The concentrations of the $Z$ complex and $Z$ axle progressively deviate more from their equilibrium values upon increasing the photon flow. Hence, we can qualitatively conclude that a larger energy input - more photons per unit time- drives the system farther away from equilibrium.

Numerical simulations, performed using experimentally determined (photo)chemical and photophysical parameters, provided time dependent concentration profiles for all species which are in excellent agreement with the experimental data (Fig. 3f-j and S4). Moreover, the simulated data also confirmed that the disassembly of $Z$ complex acts as the bottleneck for the reaction network and is thus kinetically accumulated in different amounts depending on the intensity of the incident light. 


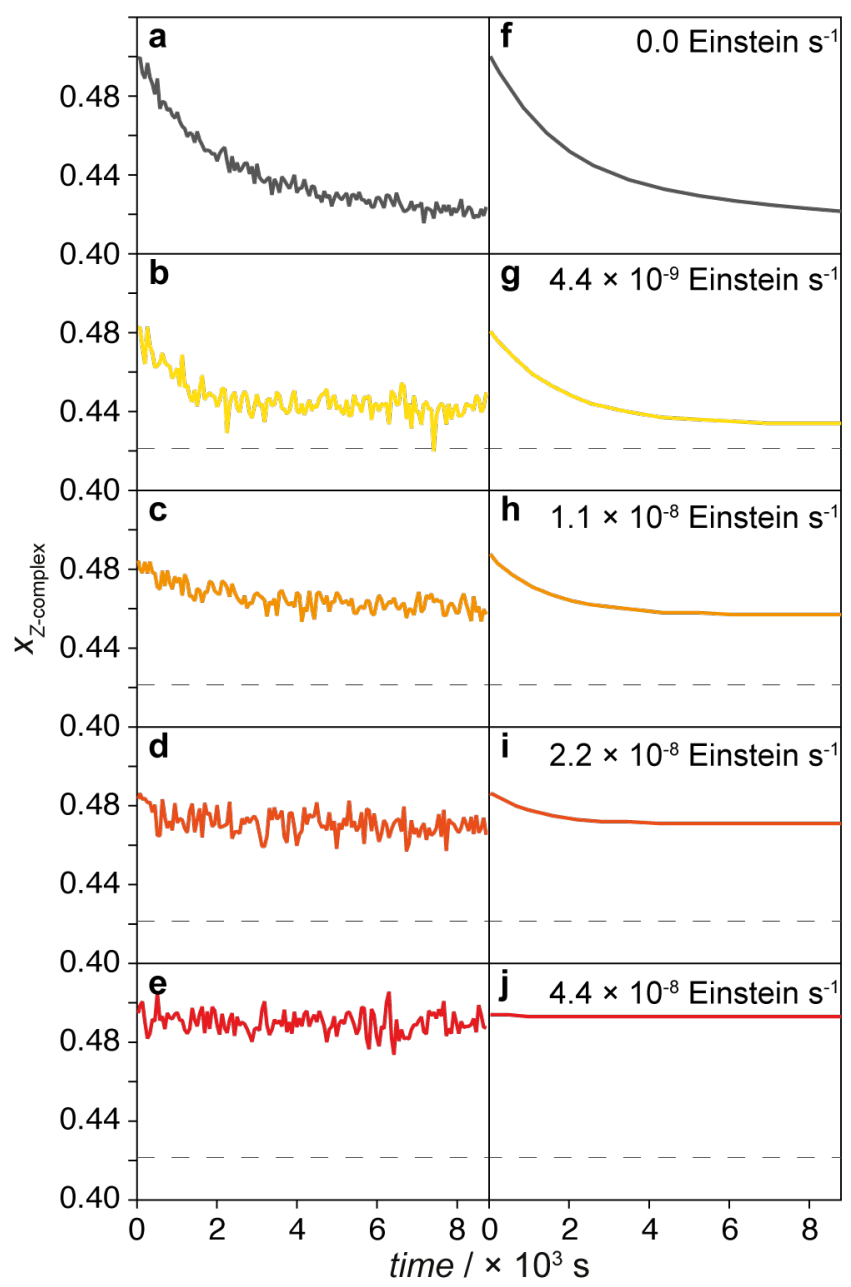

Figure 3. Time-dependent concentration profiles of complexed Z-2+. Experimental (left) and simulated (right) molar fraction profiles after photogeneration of the kinetically trapped $Z$ complex in the dark $(a, f)$ and upon constant irradiation at $365 \mathrm{~nm}$ with different light intensities (b-e and g-j). The photon flow employed to maintain the dissipative state is indicated on the right-hand side of each panel. The dashed line marks the molar fraction of the $Z$ complex at the local equilibrium in the dark (panels $a$ and $f$ ). Conditions: $\mathrm{CD}_{3} \mathrm{CN}, 298 \mathrm{~K},[1]=\left[\mathbf{2}^{+}\right]$(initial concentrations for each experiment are reported in Table S2).

\section{Kinetic and energetic considerations.}

The possibility to directly measure or estimate by means of simulations the concentration of the species at the dissipative steady state provided us the unprecedented opportunity to gain quantitative information on the reaction network under autonomous cycling away from equilibrium.

The first quantity that can be extracted from our data is the rate of cycling under irradiation. As discussed above, in a closed cycle of reactions, at the steady state, all the reactions must proceed at the same net rate. Therefore, the rate of cycling at the steady state can be calculated on any reaction of the network (Fig. 2). The rate of reaction $3\left(v_{3}\right)$ was calculated using a mixed order kinetic equation (equation 1) from the experimental concentrations at the steady state and rate constants (Table 2). 


$$
v_{3}=k_{\text {in }}^{Z}[\text { Z axle }][1]-k_{\text {out }}^{Z}[Z \text { complex }] \equiv-v_{c y}
$$

The rate $v_{3}$ is negative for all photon flows, indicating that the $Z$ complex is undergoing dethreading, and that the cycle shown in Fig. 2 is being travelled clockwise (dashed circular arrow), consistently with previous considerations on the kinetic asymmetry. As anticipated, the cycling rate $\left(v_{\mathrm{cy}}\right)$ becomes larger upon increasing the photon flow (Table 2, Fig. 4a). Finally, the $K_{\mathrm{r}}$ of the system, calculated from the simulated clockwise and anticlockwise reaction rates, is close to the estimated value of 2 (see the SI).

On the contrary, the quantum yield of the cycle $\left(\Phi_{\mathrm{cy}}\right.$, Table 2$)$, that is the number of molecules that have completed a full cycle upon the absorption of one photon, decreases from $11 \%$ at the lowest photon flow to $2 \%$ at the highest (Fig. $4 a$ ). This means that, on average at the lowest light intensity, a given ring/axle pair needs to absorb 9 photons to complete a cycle, while this number becomes more than five times larger at the highest intensity (Table 2).

The chemical potential difference $(\Delta \mu)$ of each process at the steady state can be directly calculated from the experimental or simulated concentrations (see the $\mathrm{SI}$ ) and can be interpreted as a measure of how far the reaction is from equilibrium. ${ }^{45}$ Both the steady state $\Delta \mu$ of the selfassembly of the $E$ (reaction $1, \Delta \mu_{1}$ ) and $Z$ (reaction $3, \Delta \mu_{3}$ ) complexes increase in magnitude upon increasing the photon flow (Table 2). This means that both reactions are progressively displaced farther from their equilibrium state; however, they exhibit opposite trends. Specifically, $\Delta \mu_{3}$ becomes more positive upon increasing the photon flow, showing that larger light intensities drive reaction 3 more toward the product. Such an observation is consistent with the kinetic accumulation of the $Z$ complex and with the increasingly negative values of $v_{3}$. Conversely, $\Delta \mu_{1}$ becomes more negative at higher light intensities, indicating that reaction 1 is progressively shifted back toward the reagents. At the steady state, therefore, the $Z$ complex and $E$ axle are accumulated, whereas the $E$ complex and $Z$ axle are depleted due to purely kinetic effects. ${ }^{46}$

From a thermodynamic point of view, our system can be thought of as a thermal machine powered by the gradient between the temperature $T$ of the solvent (the cold reservoir) and the temperature $T_{\mathrm{hv}}$ of the radiation (the hot reservoir), which can be correlated to the experimental photon flow using Planck's law (see the SI). As a consequence, its efficiency is limited by Carnot's theorem. ${ }^{35,36}$ The gradient of temperature powering the machine also relates to the chemical potential gradient generated by the radiation (Table S5). ${ }^{37,35,36,47}$ In the present case, the gradient is about one order of magnitude higher than that delivered by the hydrolysis of ATP in physiological conditions, and comparable to that generated by i.e. the decarboxylation of the fluorenylmethoxycarbonyl group employed to power other synthetic molecular motors. ${ }^{6}$

Each time a photon is absorbed by the system, its free energy can be either dissipated or converted into chemical free energy by changing the concentration distribution. ${ }^{45,42,37,48}$ This can be expressed with the following instantaneous free energy balance: 


$$
\dot{W}_{h v}=d_{t} G+T \dot{\Sigma}
$$

where $\dot{W}_{h v}, d_{t} G$, and $T \dot{\Sigma}$ are the free energy densities respectively absorbed, stored, and dissipated by the system per unit time. By integrating equation 2 it is possible to compute the amount of free energy dissipated and stored in any time interval (see the $\mathrm{SI}$ ).

When the system reaches the steady state, the power absorbed from the radiation is no longer stored in the system $\left(d_{t} G=0\right)$, but is entirely dissipated to sustain the non-equilibrium steady state. ${ }^{48}$ Thus, over one cycle, $\Delta_{c y} W_{h v}=T \Delta_{c y} \Sigma$. This free energy dissipation can be split into two contributions: that of the self-assembly and that of the isomerization processes, namely $T \Delta_{c y} \Sigma=T \Delta_{c y} \Sigma_{s a}+T \Delta_{c y} \Sigma_{i s o} \cdot{ }^{49}$ The self-assembly dissipation $\left(T \Delta_{c y} \Sigma_{s a}\right)$ is the portion of the absorbed free energy responsible for keeping the self-assembly steps out-of-equilibrium, and for sustaining the unidirectional motion of the rings with respect to the axles. This value can also be calculated from $\Delta \mu_{1}$ and $\Delta \mu_{3}$ (see the SI). Dissipation increases with the photon flow (Table 2), consistently with the self-assembly steps being progressively shifted away from their equilibrium state. The fraction of free energy input dissipated by the self-assembly reactions is the efficiency of free energy transduction from the photo-isomerization processes towards the self-assembly ones $\left(\eta=T \Delta_{c y} \Sigma_{s a} / \Delta_{c y} W_{h v}\right) .^{49}$ The efficiency is relatively small at all operating regime, meaning that most of the free energy harvested from the radiation is dissipated by the photoisomerization steps.

The free energy density stored in the self-assembly steps $\left(\Delta G_{s a}\right)$ coincides with the difference between the Gibbs free energy densities in the steady and local equilibrium states $\left(\Delta G_{s a}=G_{\text {light }}-G_{\text {dark }}\right.$, see the SI). Thus, it can be computed from the experimentally determined steady state concentrations. Noteworthy, the free energy storage increases with the photon flow until it reaches a plateau around $0.6 \mathrm{~J} / \mathrm{L}$. 
Table 2. Kinetic and energetic parameters of the cycle of the $1 / 2^{+}$ensemble for dissipative non-equilibrium operation under different photon flows. ${ }^{[a]}$

\begin{tabular}{cccccccc}
$\begin{array}{c}q_{\mathrm{p}} \\
\left(\text { Einstein s}^{-1}\right)^{[\mathrm{b}]}\end{array}$ & $\begin{array}{c}V_{3} \\
\left(\mu \mathrm{M} \mathrm{s}^{-1}\right)^{[\mathrm{c}]}\end{array}$ & $\begin{array}{c}\Phi_{\mathrm{cy}} \\
{[\mathrm{Nh} / \text { cycle }]^{[\mathrm{d}]}}\end{array}$ & $\begin{array}{c}\Delta \mu 1 \\
\left(\mathrm{~J} \mathrm{~mol}^{-1}\right)^{[\mathrm{e}]}\end{array}$ & $\begin{array}{c}\Delta \mu_{3} \\
\left(\mathrm{~J} \mathrm{~mol}^{-1}\right)^{[\mathrm{f}]}\end{array}$ & $\begin{array}{c}T \Delta_{c y} \Sigma_{s a} \\
\left(\mathrm{~J} \mathrm{~mol}^{-1}\right)^{[\mathrm{g}]}\end{array}$ & $\begin{array}{c}\Delta G_{s a} \\
\left(\mathrm{~J} \mathrm{~L} \mathrm{~L}^{-1}\right)^{[\mathrm{h}]}\end{array}$ & $\begin{array}{c}\eta \\
(\%)^{[\mathrm{i}]}\end{array}$ \\
\hline 0 & - & - & 0 & 0 & 0 & 0 & - \\
$4.4 \times 10^{-9}$ & -0.25 & $0.11[9]$ & -40 & 1380 & 1420 & 0.25 & 0.4 \\
$1.1 \times 10^{-8}$ & -0.34 & $0.06[16]$ & -64 & 1776 & 1840 & 0.48 & 0.3 \\
$2.2 \times 10^{-8}$ & -0.38 & $0.04[28]$ & -89 & 1961 & 2050 & 0.61 & 0.2 \\
$4.4 \times 10^{-8}$ & -0.42 & $0.02[53]$ & -149 & 1902 & 2051 & 0.63 & 0.1
\end{tabular}

[a]Conditions: $\mathrm{CD}_{3} \mathrm{CN}, 298 \mathrm{~K},[1]=\left[2^{+}\right]$(initial concentrations for each experiment are reported in Table S2), $\lambda_{\text {irr }}=365 \mathrm{~nm}$. ${ }^{[\mathrm{b}]}$ Photon flow at $365 \mathrm{~nm}$, determined by chemical actinometry. ${ }^{[\mathrm{c}]}$ Rate of reaction 3 at the stationary state; see Figure 2 for the sign convention. ${ }^{[d]}$ Quantum yield of the cycle; in brackets is the number of absorbed photons per cycle $\left(1 / \Phi_{\mathrm{cy}}\right)$. ${ }^{\mathrm{e}]}$ Residual chemical potential of reaction 1 , determined from simulated concentration values. ${ }^{[\mathrm{ff}}$ Residual chemical potential of reaction 3 , determined from experimental concentration values. ${ }^{[9]}$ Free energy dissipated by self-assembly steps during a cycle of operation at steady state. ${ }^{[\mathrm{h}]}$ Free energy density stored in the self-assembly steps at the nonequilibrium steady state. ${ }^{\left[{ }^{[} \mathrm{E}\right.} \mathrm{Energy}$ transduction efficiency, calculated as the ratio between $T \Delta_{c y} \Sigma_{s a}$ and the total amount of free energy absorbed during a cycle of operation at steady state.


Figure 4. Dependence of the kinetic and thermodynamic parameters on the photon flow. a) Net cycling rate (black dots) and quantum yield of cycling (red dots) at $298 \mathrm{~K}$. b) Free energy dissipated by the self-assembly steps during one cycle of operation at steady state. The fraction of free energy dissipated by reaction 1 (green bars) and 3 (red bars) are indicated. c) Free energy density stored in the self-assembly steps. d) Simulated forward (dark bars) and backward (light bars) rates for reactions 1 (green), 2 (purple), 3 (red), and 4 (blue) at different photon flows. In the bar graphs the photon flow is expressed as percentage of the maximum flow $\left(q_{\mathrm{n}, \mathrm{p}, \max }=4.4 \times 10^{-8}=100 \%\right)$. 
Interestingly, two distinct trends can be identified in the dependence of the determined quantities on the light intensity. Upon increasing the photon flow, $\Delta G_{\mathrm{sa}}, T \Delta_{c y} \Sigma_{s a}$, and $v_{\mathrm{cy}}$ increase, reaching a plateau between 1 and $2 \times 10^{-8}$ Einstein $\mathrm{s}^{-1}$, while $\eta$ and $\Phi$ decrease (Fig. 4a-c). This observation is in qualitative agreement with the results of stochastic simulations performed on a closely related molecular pump, which predicted that the network can process photons efficiently only as long as the photoreactions are the rate limiting steps. ${ }^{50}$ To support this claim, we compared the forward and backward rates of each reaction in the network (Fig. 4d). At the steady state, the forward and backward reactions rates (Fig. 4d) can assume different values for the four processes, provided that their relative difference is the same for all reactions. In fact, for photon flows up to $1.1 \times 10^{-8}$ Einstein $\mathrm{s}^{-1}$ (i.e., $25 \%$ of $q_{\mathrm{n}, \mathrm{p}, \max }$ ), the photochemical reactions are slower than the thermal ones and determine the overall cycling rate in either direction. Conversely, at higher flows reaction 3 becomes rate limiting (red bars in Fig. 4 d), while photoreactions proceed faster dissipating a larger fraction of light energy, thus reducing the energy transduction efficiency.

The observed decrease of the quantum yield of the cycle $\left(\Phi_{\mathrm{cy}}\right)$ (Table 2$)$ is also coherent with this picture. Notably, already at the lowest flow employed, completing one cycle requires nine photons (Table 2), a number significantly larger than the theoretical minimum value of two. This means that, in the investigated light intensity range, a given axle (complex) undergoes several $E \leftrightarrows Z$ photoisomerization events before threading (dethreading). Increasing the rate of the photoreactions increase the number of unfruitful $E \leftrightarrows Z$ isomerization events. As indicated by earlier simulations, ${ }^{50}$ only at low photon rates the pump can operate close to the maximum quantum efficiency.

\section{Conclusions}

A combination of experimental data and numerical simulations was used to characterize from a kinetic and thermodynamic point of view the dissipative photostationary states of a supramolecular pump for four intensities of incident light. Our measurements quantitatively probed the relationship between the deviation from thermodynamic equilibrium and the photon flow and provided an unprecedented insight in the non-equilibrium behaviour of (photo)chemical reaction networks. We believe that this approach is applicable to investigate any kind of light-fuelled non-equilibrium chemical ensemble, providing a testing ground for recently developed theoretical models. ${ }^{27} \mathrm{We}$ envision that our results will stimulate research on new, more sophisticated light-driven artificial molecular machines and materials capable of operating away from thermodynamic equilibrium.

\section{Methods}

Dissipative operation of the supramolecular pump. Typically, an equimolar solution of 1 and $E-2^{+}$in air-equilibrated $\mathrm{CD}_{3} \mathrm{CN}\left(8.9 \times 10^{-3} \mathrm{M}, 0.6 \mathrm{~mL}\right)$ was allowed to reach thermodynamic equilibrium in the dark at $298 \mathrm{~K}$. Subsequently a non-equilibrium mixture of the corresponding free and complexed ${\mathrm{Z}-2^{+}}^{+}$was obtained by photoisomerization of the azobenzene unit. 
Photoisomerization was performed in situ using a LED Illuminator $\left(1.5 \mathrm{~W}, \lambda_{\max }=369 \mathrm{~nm}, \mathrm{FWHM}\right.$, $15.56 \mathrm{~nm}$ ) equipped with a bandpass filter centred at $(365 \pm 5) \mathrm{nm}$. A quartz optical fibre (core $1000 \mu \mathrm{m}, 5 \mathrm{~m}$ ) was used to channel light to the solution. The terminal end of the optical fibre the quartz core was exposed and submerged into the solution within the NMR tube. Upon reaching a stable ZIE composition (PSS) irradiation was interrupted (dark, relaxation to local equilibrium) or regulated (light-on, dissipative operation) using the appropriate neutral density filter and the timedependent concentration changes of the species were followed by ${ }^{1} \mathrm{H}$ NMR.

Dynamic simulations. The linear time-variant (LTV) system of differential equation describing the pumping cycle was implemented considering three subsequent time-invariant steps (LTI): a) equilibration in the dark, b) photoisomerization, and c) operation regime. For each LTI step the reaction rates for all process and species concentrations were computed separately at any time point. The final concentrations were taken as initial conditions for the subsequent LTI step.

\section{Acknowledgements}

Financial support from the EU (H2020 ERC AdG 692981 and ERC-2015-CoG n. 681456) and the Ministero dell'Università e della Ricerca (PRIN 20173L7W8K and 201732PY3X, FARE R16S9XXKX3) is gratefully acknowledged.

\section{Author contributions}

S.C. and J.G. synthesized the compounds. M.T.B., S.C., S.S., and M.B. designed and performed the kinetic experiments and analysed the data. S.C. carried out numerical simulations. E.P. and M.E. performed the thermodynamic analysis. A.C. and S.C. wrote the manuscript with inputs from all the authors. All the authors discussed the results and commented on the manuscript. A.C. conceived the project and directed the research.

\section{References}

Schliwa, M. \& Woehlke, G. Molecular motors. Nature, 422, 759-765 (2003).

Goodsell, D. S. The machinery of life. (Copernicus, 2009).

Solis Muñana, P., Ragazzon, G., Dupont, J., Ren, C. Z.-J., Prins, L. J. \& Chen J. L.-Y. SubstrateInduced Self-Assembly of Cooperative Catalysts. Angew. Chem. Int. Ed. 57,16469-16474 (2018).

4 Yang, S., Schaeffer, G., Mattia, E., Markovitch, O., Liu, K., Hussain, A. S., Ottel, J., Sood, A. \& Otto, S. Chemical Fueling Enables Molecular Complexification of Self-Replicators. Angew. Chem. Int. Ed. 60, 11344-11349 (2021).

Boekhoven, J. et al. Dissipative self-assembly of a molecular gelator by using a chemical fuel. Angew. Chem. Int. Ed. 49, 4825-4828 (2010).

Wilson, M. R., Sola, J., Carlone, A. Goldup, S. M. Lebrasseur, N. \& Leigh, D. A. An Autonomous Chemically Fueled Small-Molecule Motor. Nature 534, 235-240 (2016).

Erbas-Cakmak, S., Fielden, S. D. P., Karaca, U., Leigh, D. A., McTernan, C. T., Tetlow, D. J. \& Wilson, M. R. Rotary and Linear Molecular Motors Driven by Pulses of a Chemical Fuel. Science 358, 340343 (2017).

Amano, S., Fielden, S. D. P. \& Leigh, D. A. A catalysis-driven artificial molecular pump. Nature 594, 529-534 (2021). 

Artificial Molecular Pump. Nat. Nanotechnol., 10, 547-553 (2015).

10 Qiu, Y. et al. A Precise Polyrotaxane Synthesizer. Science 368, 1247-1253 (2020).

11 Astumian, R. D. Optical vs. Chemical Driving for Molecular Machines. Faraday Discuss. 195, 583597 (2016).

12 Kathan, M. \& Hecht, S. Photoswitchable Molecules as Key Ingredients to Drive Systems Away from the Global Thermodynamic Minimum. Chem. Soc. Rev. 46, 5536-5550 (2017).

13 Baroncini, M., Silvi, S. \& Credi, A. Photo- and Redox-Driven Artificial Molecular Motors. Chem. Rev. 120, 200-268 (2020).

14 Feringa, B. L. The Art of Building Small: From Molecular Switches to Motors (Nobel Lecture). Angew. Chem., Int. Ed. 56, 11060-11078 (2017).

15 Ragazzon, G., Baroncini, M., Silvi, S., Venturi, M. \& Credi A. Light-powered autonomous and directional molecular motion of a dissipative self-assembling system. Nat. Nanotechnol. 2015, 10, 70-75.

16 Ceroni, P., Credi, A. \& Venturi, M. Light to investigate (read) and operate (write) molecular devices and machines. Chem. Soc. Rev. 43, 4068-4083 (2014).

17 Foy, J. T., Li, Q., Goujon, A., Colard-Itté, J.-R., Fuks, G., Moulin, E., Schiffmann, O., Dattler, D., Funeriu, D. P. \& Giuseppone, N. Dual-Light Control of Nanomachines that Integrate Motor and Modulator Subunits. Nat. Nanotechnol. 12, 540-545 (2017).

18 T. Ikegami, Y. Kageyama, K. Obara, S. Takeda, Dissipative and Autonomous Square-Wave SelfOscillation of a Macroscopic Hybrid Self-Assembly under Continuous Light Irradiation. Angew. Chem. Int. Ed. 55, 8239-8243 (2016).

19 Herder, M. \& Lehn J.-M. The Photodynamic Covalent Bond: Sensitized Alkoxyamines as a Tool To Shift Reaction Networks Out-of-Equilibrium Using Light Energy. J. Am. Chem. Soc. 140, 7647-7657 (2018).

20 Greb, L., Eichhçfer, A. \& Lehn, J.-M. Synthetic Molecular Motors: Thermal N Inversion and Directional Photoinduced $\mathrm{C}=\mathrm{N}$ Bond Rotation of Camphorquinone Imines. Angew. Chem. Int. Ed. 54, 14345 14348 (2015).

21 Cheng, C., McGonigal, P. R., Stoddart, J. F. \& Astumian, R. D. Design and Synthesis of Nonequilibrium Systems, ACS Nano 9, 8672-8688 (2015).

22 Merindol, R. \& Walther, A. Materials Learning from Life: Concepts for Active, Adaptive and Autonomous Molecular Systems. Chem. Soc. Rev. 46, 5588-5619 (2017).

23 G. Ragazzon, L. J. Prins, Energy Consumption in Chemical Fuel-Driven Self-Assembly. Nat. Nanotechnol. 13, 882-889 (2018).

24 van Rossum, S. A. P., Tena-Solsona, M., van Esch, J. H., Eelkema, R. \& Boekhoven, J. Dissipative Out-of-Equilibrium Assembly of Man-Made Supramolecular Materials. Chem. Soc. Rev. 46, 55195535 (2017).

25 Das, K., Gabrielli, L. \& Prins, L. J. Chemically Fueled Self-Assembly in Biology and Chemistry. Angew. Chem. Int. Ed. 60,20120-20143 (2021).

26 Mattia, E. \& Otto, S. Supramolecular systems chemistry. Nat. Nanotechnol. 10, 111-119 (2015).

27 Sorrenti, A., Leira-Iglesias, J., Sato, A. \& Hermans, T. M. Non-equilibrium steady states in supramolecular polymerization. Nat. Commun. 8, 15899-1-8 (2017).

28 Geertsema, E. M., van der Molen, S. J., Martens, M. \& Feringa, B. L. Optimizing rotary processes in synthetic molecular motors. Proc. Nat. Acad. Sci. USA 106, 16919-16924 (2009).

29 Wilcken, R., Gerwien, A., Huber, L. A., Dube, H. \& Riedle, E. Quantitative in-situ NMR-Illumination for Excitation and Kinetic Analysis of Molecular Motor Intermediates. ChemPhotoChem

30 Corra, S., Casimiro, L., Baroncini, M., Groppi, J., La Rosa, M., Tranfić Bakić, M., Silvi, S. \& Credi, A. Artificial Supramolecular Pumps Powered by Light. Chem. Eur. J. 27, 11076-11083 (2021).

31 Canton, M., Groppi, J., Casimiro, L., Corra, S., Baroncini, M., Silvi, S. \& Credi, A. Second-Generation Light-Fueled Supramolecular Pump. J. Am. Chem. Soc. 143, 10890-10894 (2021).

32 Nitschke, P., Lokesh, N. \& Gschwind, R. M. Combination of illumination and high resolution NMR spectroscopy: Key features and practical aspects, photochemical applications, and new concepts. Prog. Nucl. Magn. Reson. Spectrosc. 2019, 114, 86-134.

33 Baroncini, M., Silvi, S., Venturi, M. \& Credi, A. Reversible Photoswitching of Rotaxane Character and Interplay of Thermodynamic Stability and Kinetic Lability in a Self-Assembling Ring-Axle Molecular System. Chem. Eur. J. 2010, 16, 11580-11587.

34 Blackmond, D. G. "If Pigs Could Fly" Chemistry: A Tutorial on the Principle of Microscopic Reversibility Angew. Chem. Int. Ed. 48, 2648-2654 (2009).

35 Ross, R. T. Some Thermodynamics of Photochemical Systems. J. Chem. Phys. 45, 1-7 (1966).

36 Porter, G. Transfer and Storage of Chemical and Radiation Potential. J. Chem. Soc. Faraday Trans. 2 79, 473-482 (1983).

37 Penocchio, E., Rao, R. \& Esposito, M. Nonequilibrium Thermodynamics of Light-Induced Reactions. J. Chem. Phys. 155, 114101 (2021). 
38 The fictitious "equilibrium constants" for the photochemical steps are defined as the ratio of the concentrations of the products and reactants at the PSS for the respective reactions.

39 Onsager, L. Reciprocal Relations in Irreversible Processes. I. Phys. Rev. 37, 405-426 (1931).

40 Astumian, R. D. \& Bier, M. Mechanochemical Coupling of the Motion of Molecular Motors to ATP Hydrolysis. Biophys. J. 70, 637-653 (1996).

41 Astumian, R. D. Stochastic Conformational Pumping: A Mechanism for Free-Energy Transduction by Molecules. Annu. Rev. Biophys. 40, 289-313 (2011).

42 Kondepudi, D. \& Prigogine, I. Modern Thermodynamics: From Heat Engines to Dissipative Structures (Wiley, 1998).

43 Rao, R. \& Esposito M. Nonequilibrium Thermodynamics of Chemical Reaction Networks: Wisdom from Stochastic Thermodynamics. Phys. Rev. X 6, 041064 (2017).

44 It should be noted that the $Z$ species are kinetically trapped with respect to azobenzene isomerization, because the thermodynamically stable isomer is $E$. For the sake of simplicity, from this point onward we shall refer to the kinetically trapped state of the system as an "equilibrium state" referring to the local equilibrium of the $Z$ complex self-assembly reaction. It has to be made clear, though, that the true equilibrium state of our system is the absence of $Z$ species.

45 Demirel, Y. \& Gerbaud, V. Nonequilibrium Thermodynamics Transport and Rate Processes in Physical, Chemical and Biological Systems (Elsevier, 2019).

46 J.-M. Lehn Perspectives in Chemistry-Aspects of Adaptive Chemistry and Materials. Angew. Chem. Int. Ed. 54, 3276-3289 (2015).

47 Ries, H. \& McEvoy, A. J. "Chemical potential and temperature of light," J. Photochem. Photobiol. A 59, 11-18 (1991).

48 Penocchio, E., Rao, R. \& Esposito, M. Thermodynamic Efficiency of Dissipative Chemistry. Nat. Commun. 10, 3865 (2019).

49 Amano, S., Esposito, M. Kreidt, E., Leigh, D. A., Penocchio, E. \& Roberts, B. M. W. Insights from an Information Thermodynamics Analysis of a Synthetic Molecular Motor. https://chemrxiv.org/engage/chemrxiv/article-details/60f69064393cc9882f4db6bf (2021).

50 Sabatino, A., Penocchio, E., Ragazzon, G., Credi, A. \& Frezzato, D. Individual-Molecule Perspective Analysis of Chemical Reaction Networks: The Case of a Light-Driven Supramolecular Pump. Angew. Chem. Int. Ed. 58, 14341-14348 (2019). 